\title{
THE TYPES OF PROCTOTRUPOIDEA (HYMENOPTERA) IN THE CHARLES T. BRUES COLLECTION AT THE MUSEUM OF COMPARATIVE ZOOLOGY
}

\author{
By Lubomir MasNer* \\ Institute of Entomology, Czechoslovak Academy of Sciences, Prague
}

During a two week visit to the Museum of Comparative Zoology in June, 1964, I examined the types of 60 species of Proctotrupoidea described by Charles T. Brues. These belonged to the following families: 21 Proctotrupidae, 7 Diapriidae, 13 Scelionidae, I5 Platygasteridae, and 4 Ceraphronidae. In the present report a number of lectotypes are selected, some new combinations are suggested, and an account of the state of preservation of all types listed is given. The names and synonymy used in this paper are those proposed by Masner and Muesebeck (Supplement 2, Hymenoptera of America north of Mexico. United States Department of Agriculture, in press). The taxonomic categories subfamily and tribe are not used in the present paper; the genera and species are arranged alphabetically throughout the families. Under each species are listed the various labels on the type specimen, from top to bottom, each "label" being separated by a semicolon. Specimens are glued to card points except when specifically mentioned as pinned (on minuten nadeln).

\section{Family PROCTOTRUPIDAE}

\section{Codrus Panzer, I80r \\ Codrus angusticeps (Brues)}

Proctotrypes angusticeps Brues, 1910. Wis. Nat. Hist. Soc. Bul. 7: 112.

Mt. Constitution, Jul. 09, Wash.; Type; MCZ type label; Exallonyx angusticeps Brues

Holotype female No. 31002, well preserved. Unique.

Codrus ashmeadi (Brues)

Exallonyx ashmeadi Brues, 1919. N. Y. Ent. Soc. Jour. 27: 10, 13.

\footnotetext{
*Postdoctorate Fellow, National Research Council of Canada, Research Institute, Canada Department of Agriculture, Belleville, Ont., Canada.

Thanks are extended to the staff of the Museum of Comparative Zoology, particularly to Professor E. Mayr, Dr. H. E. Evans, and Professor P. J. Darlington, Jr. Thanks are also due to the National Research Council of Canada for support during completion of this paper.

Manuscript received by the editor January 16, 1966.
} 
Machias, Me., VII-20-09, C. W.; MCZ type label; Exallonyx ashmeadi Brues

Holotype male No. 31006, well preserved. Three paratype males. Codrus carinatus (Brues)

Exallonyx carinatus Brues, 1919. N. Y. Ent. Soc. Jour. 27: 11, 15.

Oroville, Wash., May 1, 1912; Type; MCZ type label; Exallonyx carinatus Brues

Holotype male No. 30993, well preserved. Unique.

$$
\text { Codrus fuscicornis (Brues) }
$$

Exallonyx fuscicornis Brues 1919. N. Y. Ent. Soc. Jour. 27: 10, 12.

Woods Hole, Mass.; Type; MCZ type label; Exallonyx fuscicornis Brues

Holotype male No. 3IOO5, well preserved. Unique.

$$
\text { Codrus grandis (Brues) }
$$

Exallonyx grandis Brues, 1919. N. Y. Ent. Soc. Jour. 27: 11, 17.

Ramsey, N. J.; VIII-22-9; Type; MCZ type label; Exallonyx grandis Brues

Holotype female No. 3 Ioro, well preserved. Unique.

\section{Codrus obscuripes (Brues)}

Proctotrypes obscuripes Brues, 1910. Wis. Nat. Hist. Soc. Bul. 7: 112, 114.

Mt. Constitution, Jul. 09, Wash.; Type; MCZ type label; Exallonyx obscuripes Brues

Holotype male No. 3IOO7, well preserved. One paratype male + documentary specimens.

\section{Codrus pallidicornis (Brues)}

Exallonyx pallidicornis Brues, 1919. N. Y. Ent. Soc. Jour. 27: 11, 14.

Putnam Ct., 12 July 1905, HL Viereck; Type; MCZ type label; Exallonyx pallidicornis Brues

Holotype male No. 30994, well preserved. Two paratype males.

$$
\text { Codrus parvulus (Brues) }
$$

Exallonyx parvulus Brues, 1919. N. Y. Ent. Soc. Jour. 27: 11, 16.

Oroville, Wash., I-IV, Melander; Type; MCZ type label; Exallonyx parvulus Brues

Holotype male No. 30992, left antenna after 9th segment off. Eleven paratype males.

$$
\text { Codrus placidus (Brues) }
$$

Proctotrypes placidus Brues, 1910. Wis. Nat. Hist. Soc. Bul. 7: 112, 113.

Mt. Constitution, Jul. 31-8, Wash.; Type; MCZ type label; Exallonyx placidus Brues

Holotype male No. 3IOO4, well preserved. Unique.

Codrus pleuralis (Brues)

Exallonyx pleuralis Brues, 1919. N. Y. Ent. Soc. Jour. 27: 10, 14.

Type; MCZ type label; Exallonyx pleuralis Brues

Holotype male No. 3099I, well preserved. Two paratype males. Codrus serricornis (Brues)

Proctotrypes serricornis Brues, 1910. Wis. Nat. Hist. Soc. Bul. $7: 112,115$.

Puget Sound, Wash., Jul. 08; Type; MCZ type label; Exallonyx serricornis Brues 
Holotype male No. 3I009, right antenna after 6th segment and left antenna after 3 rd segment missing. Two paratype males.

Codrus similis (Brues)

Exallonyx similis Brues, 1919. N. Y. Ent. Soc. Jour. 27: 10, 11.

Tacoma, Wash., 28-VIII-11; Type; MCZ type label; Exallonyx similis Brues

Holotype female No. 3I003. Four paratype females.

Codrus simplicior (Brues)

Proctotrypes simplicior Brues, 1910. Wis. Nat. Hist. Soc. Bul. 7: 112, 116. Brues

Puget Sound, Wash., Jul. 08; Type; MCZ type label; Exallonyx simplicior

Holotype male No. 30990, right antenna after 7 th segment missing, left antenna and wings missing, legs and gaster glued separately to card point. Unique (!).

\section{Cryptoserphus Kieffer, I907}

Cryptoserphus melanderi Brues

Cryptoserphus melanderi Brues, 1919. N. Y. Ent. Soc. Jour. 27: 8.

Pullman, Wash., V-18-09; Type; MCZ type label; Cryptoserphus melanderi Brues

Holotype male No. 3100o, well preserved. Two paratype males.

Cryptoserphus occidentalis Brues

Cryptoserphus occidentalis Brues, 1919. N. Y. Ent. Soc. Jour. 27: 7.

Chatcolet Lake, Idaho, VIII-1915, A. L. Melander; Type; MCZ type label; Cryptoserphus occidentalis Brues

Holotype female No. 30999, left antenna missing after 2nd segment. Four paratype females.

\section{Nothoserphus Brues, I940 \\ Nothoserphus mirabilis Brues}

Notroserphus mirabilis Brues, 1940. Amer. Acad. Arts Sci. Proc. 73: 263.

Taihorin, Formosa, H. Sauter, 1911; 7. VII; MCZ type label; Nothoserphus mirabilis Brues

Holotype male No. 29789, left antenna broken after 3rd segment, but remainder glued to mount; right antenna missing after 9th segment (pinned). One paratype male.

\section{Phaenoserphus Kieffer, I908}

Phaenoserphus longipes Brues

Phaenoserphus longipes Brues, 1919. N. Y. Ent. Soc. Jour. 27: 9.

Almota, Wash., 24-VI, Melander; Type; MCZ type label; Phaenoserphus longipes Brues

Holotype male No. 3IоOI, well preserved. Unique.

$$
\begin{aligned}
& \text { Proctotrupes Latreille, I796 } \\
& \text { Proctotrupes cockerelli (Brues) }
\end{aligned}
$$

Serphus cockerelli Brues, 1919. N. Y. Ent. Soc. Jour. 27: 3, 4.

Eldora, Colo., Aug. 18 (Cockerell); Type; MCZ type label; Serphus cockerelli Brues

Holotype male No. 30997. well preserved (pinned). Unique. 


\section{Proctotrupes debilis (Brues)}

Serphus debilis Brues, 1919. N. Y. Ent. Soc. Jour. 27: 3, 5.

Wawai, Wash., 20-V-11; Type; MCZ type label; Serphus debilis Brues

Holotype male No. 30998, well preserved. Unique.

Proctotrupes sequoiarum (Brues)

Serphus sequoiarum Brues, 1919. N. Y. Ent. Soc. Jour. 27: 3, 4.

Muir Woods, VIII-7-15, Cal., A. L. Melander; Type; MCZ type label; Serphus sequoiarum Brues

Holotype male No. 30996, left antenna after 8th segment off, left front leg after coxa missing. Unique.

\section{Proctotrupes zabriskiei (Brues)}

Serphus zabriskiei Brues, 1919. N. Y. Ent. Soc. Jour. 27: 2, 3.

Rochester, N. Y., J. L. Zabriskie, 10. VI. 1905; Type; MCZ type label; Serphus zabriskiei Brues

Holotype female No. 30995, left antenna after 8th segment off. Unique.

\section{Family DIAPRIIDAE}

Auxopaedeutes Brues, 1903

Auxopaedeutes lyriformis Brues

Auxopaedeutes lyriformis Brues, 1910. Wis. Nat. Hist. Soc. Bul. 8: 82.

Forest Hills, Mass., V-16-1910; MCZ type label; Auxopaedeutes lyriformis Brues Type

Holotype female No. 31027, well preserved. Unique.

Entomacis Foerster, I 856

Entomacis ambigua (Brues)

Hemilexodes ambigua Brues, 1903. Amer. Ent. Soc. Trans. 29: 126.

Nest Stenamma fulvum piceum, Colebrook, 8.25.01; Type; MCZ type label; lectotype selected by L. Masner, 1964; Hemilexodes ambigua Brues

Lectotype female No. 3ro2I, well preserved. Two additional females (paralectotypes).

Hoplopria Ashmead, I893

Hoplopria grandis Brues

Hoplopria grandis Brues, 1915. Psyche 22: 11.

Porto Velho, Rio Madeira, Brazil, Mann \& Baker; Type; MCZ type label; Hoplopria grandis Brues of type

Holotype female No. 31022, well preserved. Unique.

$$
\text { Psilus Panzer, I80 I }
$$

Psilus debilis (Brues) comb. $\mathrm{n}$.

Galesus debilis Brues, 1915. Psyche 22: 12.

Independencia, Parahyba, Brazil, Mann \& Heath; Type; MCZ type label; Galesus debilis Brues of type

Holotype female No. 31025, well preserved. Unique.

Trichopria Ashmead, I 893

Trichopria acutiventris (Brues)

Phaenopria acutiventris Brues, 1902. Amer. Nat. 36: 372. 
Austin, Tex., 3.01; Type; MCZ type label; in nest Eciton caecum; Phaenopria acutiventris Brues

Holotype female No. 3IO26, well preserved. Unique.

Xanthopria Brues, I9I5

Xanthopria nitida Brues

Xanthopria nitida Brues, 1915. Psyche 22: 11.

Independencia, Parahyba, Brazil, Mann \& Heath; Type; MCZ type label; Xanthopria nitida Brues of type

Holotype female No. 3IO24, well preserved. Two paratype females (!).

Yanthopria opaca Brues

Xanthopria opaca Brues, 1915. Psyche 22: 10.

Independencia, Parahyba, Brazil, Mann \& Heath; Type; MCZ type label; Xanthopria opaca Brues of type

Holotype female No. 3IO23, well preserved. Two paratype females.

\section{Family SCELIONIDAE}

Calotelea Westwood, 1837

Calotelea parvipennis Melander et Brues

Caloteleia (!) parvipennis Melander et Brues, 1903. Biol. Bul. 5: 22.

Woods Hole, Mass.; Halictus; Type; MCZ type label; Caloteleia parvipennis Melander et Brues type

Holotype female No. 3ror4, right antenna after 7 th segment and left antenna after 6 th segment off. Unique (!).

$$
\begin{gathered}
\text { Gryon Haliday, I } 833 \\
\text { Gryon robustus (Brues) }
\end{gathered}
$$

Hadronotus robustus Brues, 1907. Wis. Nat. Hist. Soc. Bul. 5: 156.

Austin, Tex.; Type; MCZ type label; Hadronotus robustus Brues

Holotype female No. 3IOI7, well preserved. Unique.

$$
\begin{aligned}
& \text { Lepidoscelio Kieffer, I905 } \\
& \text { Lepidoscelio viatrix Brues }
\end{aligned}
$$

Lepidoscelio viatrix Brues, 1917. Nat. Acad. Sci. Proc. 3 : 139.

MCZ type label; Lepidoscelio viatrix Brues, type

Holotype female No. 3ior8. Two paratype females ("On an Orthacris sp. det Uvarov").

\section{Macroteleia Westwood, I 835}

Macroteleia caerulea (Brues) comb. n.

Cacellus caeruleus Brues, 1918. Mus. Comp. Zool. Harv. Bul. 62: 122.

Graciosa Bay, Santa Cruz Is., W. M. Mann '16; MCZ type label; Cacellus caeruleus Brues

Holotype female No. 9070, well preserved. Unique.

Classified in Macroteleia with some doubts.

Macroteleia herbigrada Brues

Macroteleia herbigrada Brues, 1915. Psyche 22: 7. 
Independencia, Parahyba, Brazil, Mann and Heath; Type; MCZ type label; Macroteleia herbigrada Brues, type $\hat{o}$

Holotype male No. 3I016, right antenna after 6th segment off. Unique.

Macroteleia pallipes (Brues) comb. n.

Hoploteleia pallipes Brues, 1915. Psyche 22: 8.

Para, Brazil, W. M. Mann; Type; MCZ type label; Hoploteleia pallipes Brues, type ô

Holotype male No. 3IOI3, well preserved. Unique.

Classified in Macroteleia Westwood with some doubts.

Paridris Kieffer, I908

Paridris nigricornis (Brues)

Idris nigricornis Brues, 1903. Amer. Ent. Soc. Trans. 29: 125.

Mixed nest Myr. Lep., Colebrook, 9-10-01; MCZ type label; lectotype selected by L. Masner, 1964; Idris nigricornis Brues, types (!).

Lectotype male No. 3IOI6, left antenna after 8th segment off. Two paralectotype males.

$$
\begin{aligned}
& \text { Scelio Latreille, I } 805 \\
& \text { Scelio nitens Brues }
\end{aligned}
$$

Scelio nitens Brues, 1906. Wis. Nat. Hist. Soc. Bul. 4: 110.

Type; Algoa Bay, Capland, 29.11.96, Dr. H. Brauns; MCZ type label; lectotype selected by L. Masner, 1964; Scelio nitens Brues

Lectotype female No. 3IOI9, well preserved. Two paralectotype females (!) (? conspecific with lectotype).

\section{Scelio pulchripennis Brues}

Scelio pulchripennis Brues, 1906. Wis. Nat. Hist. Soc. Bul. 4: 109.

Type: Algoa Bay, Capland, 22.1.96, Dr. Brauns; MCZ type label; Scelio pulchripennis Brues

Holotype female No. 3IO2O, well preserved. Unique (!).

$$
\text { Scelio setiger Brues }
$$

Scelio setiger Brues, 1918. Mus. Comp. Zool. Harv. Bul. 62: 121.

Ugi, British Solomon Is., W. M. Mann; MCZ type label; Scelio setiger Brues

Holotype female No. 9069, well preserved.

$$
\begin{gathered}
\text { Telenomus Haliday, I } 833 \\
\text { Telenomus fiskei Brues }
\end{gathered}
$$

Telenomus fiskei Brues, 1910. Psyche 17: 106.

Machias, Me., Aug. 20, 1909; Type; MCZ type label; Telenomus fiskei Brues

Holotype female No. 3 ror I, right antenna missing. Unique (!). Telenomus heracleicola Brues

Telenomus heracleicola Brues, 1906. Wis. Nat. Hist. Soc. Bul. 45: 143, 146.

Wash. Exp. Sta. No. 026; Type; MCZ type label; lectotype selected by L. Masner, 1964; Telenomus heracleicola Brues

Lectotype female No. 3IOI2, well preserved (pinned). One paralectotype female (!). 


\section{Trimorus Foerster, I 856 \\ Trimorus fortis (Brues) comb. $\mathrm{n}$.}

Hoplogryon fortis Brues, 1915. Psyche 22: 6.

Independencia, Parahyba, Brazil, Mann \& Heath; Type; MCZ type label; Hoplogryon fortis Brues ô type

Holotype male No. 30987, well preserved. Unique.

\section{Family PLATYGASTERIDAE}

Fidiobia Ashmead, I 895

Fidiobia tritici (Brues)

Rosneta tritici Brues, 1908 Wis. Nat. Hist. Soc. Bul. 6: 157.

Middleport, N Y., 1908; Type; MCZ type label; Rosneta tritici Brues

Holotype female No. 300, missing from the card point (fore wing only left). Unique.

\section{Gastrotrypes Brues, 1922 \\ Gastrotrypes caudatus Brues}

Gastrotrypes caudatus Brues, 1922. Amer. Acad. Arts Sci. Proc. 57: 272.

Forest Hills, Mass., 5,1916; MCZ type label; lectotype selected by L. Masner, 1964; Gastrotrypes caudatus Brues, types (!)

Lectotype female No. 3104I, well preserved. I6 paralectotype females.

\section{Gastrotrypes spatulatus Brues}

Gastrotrypes spatulatus Brues, 1922. Amer. Acad. Arts Sci. Proc. 57: 271.

Kartabo, B. G., Jul. Aug. 1920, W. M. Wheeler; MCZ type label; lectotype selected by L. Masner, 1964; Gastrotrypes spatulatus Brues

Lectotype female No. 3I040, well preserved. Two paralectotype females.

\section{Isostasius Foerster, I 856 \\ Isostasius crassus Brues}

Isostasius crassus Brues, 1922. Amer. Acad. Arts Sci. Proc. 57: 277.

Kartabo. B. G., Jul. Aug. 1920, W. M. Wheeler; MCZ type label; Isostasius crassus Brues, type

Holotype female No. 31030, well preserved. Two paratype females.

$$
\begin{gathered}
\text { Platygaster Latreille, I809 } \\
\text { Platygaster anormis (Brues) }
\end{gathered}
$$

Aneuron anormis Brues, 1910. Wis. Nat. Hist. Soc. Bul. 8 : 49.

Albany, N. Y., IV-18, 1908; MCZ type label; lectotype selected by L. Masner, 1964; Aneuron anormis Brues

Lectotype male No. 3ro39, left antenna after 4 th segment missing. Four paralectotype males and one paralectotype female.

$$
\text { Platygaster carinifrons (Brues) comb. } \mathrm{n} \text {. }
$$

Polygnotus carinifrons Brues, 1910. Broteria 9: 155.

MCZ type label; lectotype selected by L. Masner, 1964; Polygnotus carinifrons Brues 
Lectotype male (!) No. 3IO34, well preserved. Three paralectotype males but no female.

Platygaster coronatus (Brues) comb. n.

Polygnotus coronatus Brues, 1910. Broteria 9: 154.

MCZ type label; lectotype selected by L. Masner, 1964; Polygnotus coronatus Brues

Lectotype female No. 3I035, well preserved. Three paralectotype males.

Platygaster latescens (Brues) comb. n.

Polygnotus latescens Brues, 1910. Broteria 9: 156.

MCZ type label; lectotype selected by L. Masner, 1964; Polygnotus latescens Brues

Lectotype female No. 31036, well preserved. Three paralectotype females.

Platygaster mediocris (Brues) comb. $\mathrm{n}$.

Polygnotus mediocris Brues, 1910. Broteria 9: 157.

MCZ type label; lectotype selected by L. Masner, 1964; Polygnotus mediocris Brues

Lectotype female No. 31037, well preserved. Three paralectotype males.

\section{Platygaster noveboracensis (Brues)}

Eritrissomerus noveboracensis Brues, 1910. Wis. Nat. Hist. Soc. Bul. 8 : 48.

Albany, N. Y., IV. 5, 1907; Type; MCZ type label; lectotype selected by L. Masner, 1964; Eritrissomerus noveboracensis Brues, types (!)

Lectotype male No. 3IO38, well preserved. Two paralectotype males.

Platygaster simplex (Brues) comb. n.

Polygnotus simplex Brues, 1922. Amer. Acad. Arts Sci. Proc. 57: 257.

\# b; MCZ type label; lectotype selected by L. Masner, 1964; Polygnotus simplex Brues

Lectotype female No. 31032, well preserved. Two paralectotype females (!).

\section{Platygaster tubulosa Brues}

Platygaster tubulosa Brues, 1922. Amer. Acad. Arts Sci. Proc. 57: 276.

MCZ type label; Platygaster tubulosa Brues, type

Holotype female No. 3IO33, well preserved. Two paratype females.

\section{Sceliotrachelus Brues, I908}

Sceliotrachelus braunsi Brues

Sceliotrachelus braunsi Brues, 1908. Genera Insectorum, fasc. 80: 13.

Algoa Bay, Capland, Dr. Brauns; MCZ type label; Sceliotrachelus braunsi Brues, type

Holotype male (!) No. 30989, right antenna after 7 th segment off (pinned). One paratype male. 
Synopeas Foerster, I 856

Synopeas minor (Brues)

Polymecus (Dolichotrypes) minor Brues, 1922. Amer. Acad. Arts Sci. Proc. 57: 268.

Kartabo, B. G., Jul. Aug. 1920, W. M. Wheeler; MCZ type label; lectotype selected by L. Masner, 1964; Polymecus (Dolichotrypes) minor Brues 우 우

Lectotype female No. 3I028, well preserved. Two paralectotype females (!).

Trichacis Foerster, I 856

Trichacis meridionalis (Brues) comb. $\mathrm{n}$.

Xestonotus meridionalis Brues, 1910. Broteria 9: 150.

MCZ type label; lectotype selected by L. Masner, 1964; Xestonotus meridionalis Brues; Trichacis (Fouts)

Lectotype female No. 3IO3I, well preserved. One paralectotype female (!).

\section{Family CERAPHRONIDAE \\ Ceraphron Jurine, I807 Ceraphron croceipes Brues}

Ceraphron croceipes Brues, 1902. Amer. Nat. 36:369.

Type; in nest $E$. coecum; $\mathrm{MCZ}$ type label; Ceraphron croceipes Brues Holotype female No. 30984, well preserved. Unique.

$$
\begin{gathered}
\text { Conostigmus Dahlbom, I } 858 \\
\text { Conostigmus orcasensis (Brues) }
\end{gathered}
$$

Megaspilus orcasensis Brues, 1910. Wis. Nat. Hist. Soc. Bul. 7: 118

Puget Sound, Wash., Jul. 08; MCZ type label; Megaspilus orcasensis Brues, ô type

Holotype male No. 30985, well preserved. Unique.

\section{Ecitonetes Brues, 1902}

Ecitonetes subapterus Brues

Ecitonetes subapterus Brues, 1902. Amer. Nat. 36: 370.

Type; Austin, Tex.; MCZ type label; Ecitonetes subapterus Brues

Holotype female No. 30983, thorax slightly cracked. Unique.

Lygocerus Foerster, I 856

Lygocerus constrictus Brues

Lygocerus constrictus Brues, 1910. Wis. Nat. Hist. Soc. Bul. 7: 119.

Mt. Constitution, Jul. 09, Wash.; MCZ type label; Lygocerus constrictus Brues, type

Holotype male No. 30986, well preserved. Two paratype males. 


\section{List of the types of Proctotrupoidea}

acutiventris, Trichopria, 298 ambigua, Entomacis, 298 angusticeps, Codrus, 295 anormis, Platygaster, 301 ashmeadi, Codrus, 295 braunsi, Sceliotrachelus, 302 caerulea, Macroteleia, 299 carinatus, Codrus, 296 carinifrons, Platygaster, 301 caudatus, Gastrotrypes, 301 cockerelli, Proctotrupes, 297 constrictus, Lygocerus, 303 coronatus, Platygaster, 302 crassus, Isostasius, 301 croceipes, Ceraphron, 303 debilis, Proctotrupes, 298 debilis, Psilus, 298 fiskei, Telenomus, 300 fortis, Trimorus, 301 fuscicornis, Codrus, 296 grandis, Codrus, 296 grandis, Hoplopria, 298 heracleicola, Telenomus, 300 herbigrada, Macroteleia, 299 latescens, Platygaster, 302 longipes, Phaenoserphus, 297 lyriformis, Auxopaedeutes, 298 mediocris, Platygaster, 302 melanderi, Cryptoserphus, 297 meridionalis, Trichacis, 303 minor, Synopeas, 303

mirabilis, Nothoserphus, 297

nigricornis, Paridris, 300

nitens, Scelio, 300

nitida, Xanthopria, 299

noveboracensis, Platygaster, 302

occidentalis, Cryptoserphus, 297

obscuripes, Codrus, 296

opaca, Xanthopria, 299

orcasensis, Conostigmus, 303

pallidicornis, Codrus, 296

pallipes, Macroteleia, 300

parvipennis, Calotelea, 299

parvulus, Codrus, 296

placidus, Codrus, 296

pleuralis, Codrus, 296

pulchripennis, Scelio, 300

robustus, Gryon, 299

serricornis, Codrus, 296

sequoiarum, Procotrupes, 298

setiger, Scelio, 300

similis, Codrus, 297

simplex, Platygaster, 302

simplicior, Codrus, 297

spatulatus, Gastrotrypes, 301

subapterus, Ecitonetes, 303

tritici, Fidiobia, 301

tubulosa, Platygaster, 302

viatrix, Lepidoscelio, 299

zabriskiei, Proctotrupes, 298 

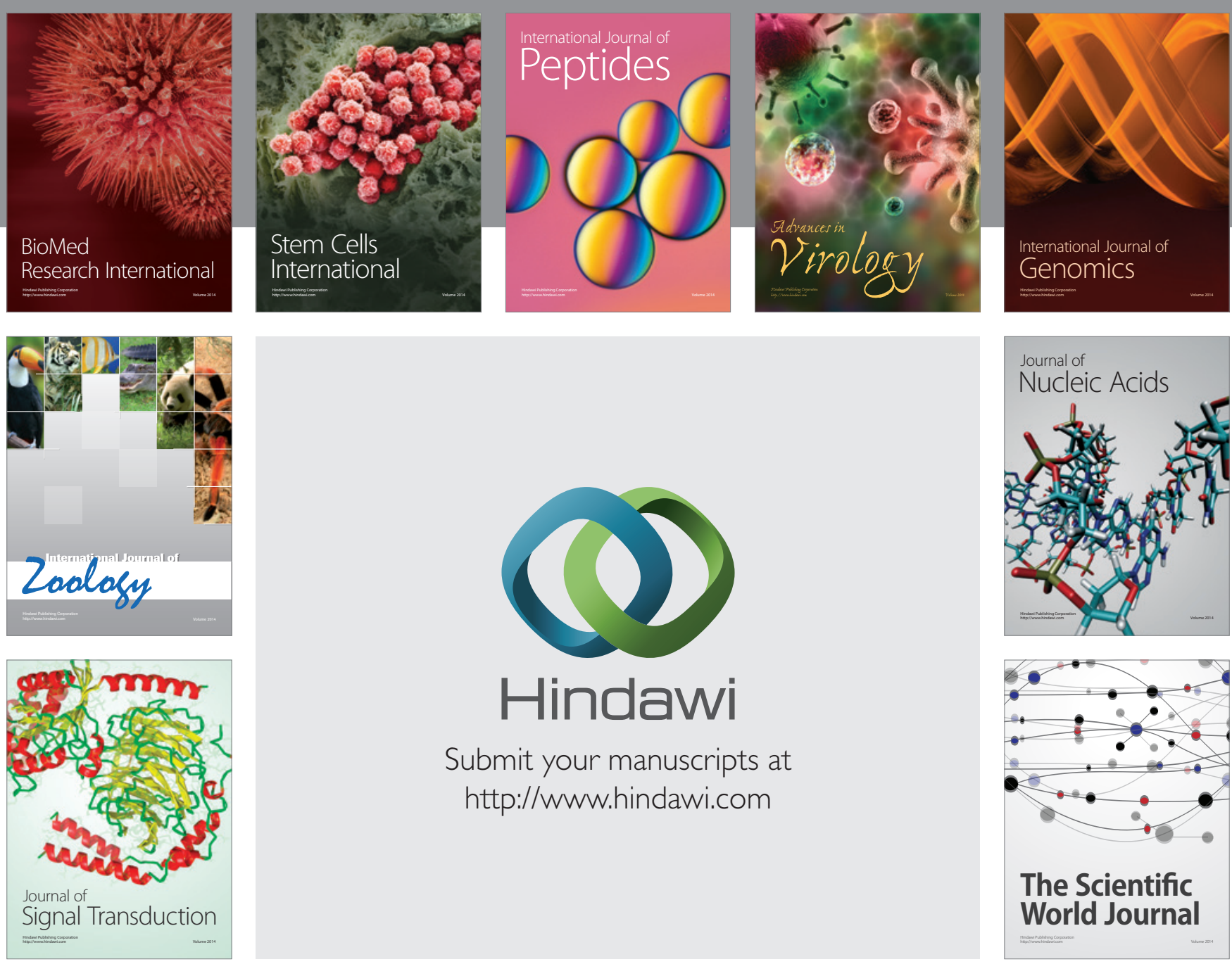

Submit your manuscripts at

http://www.hindawi.com
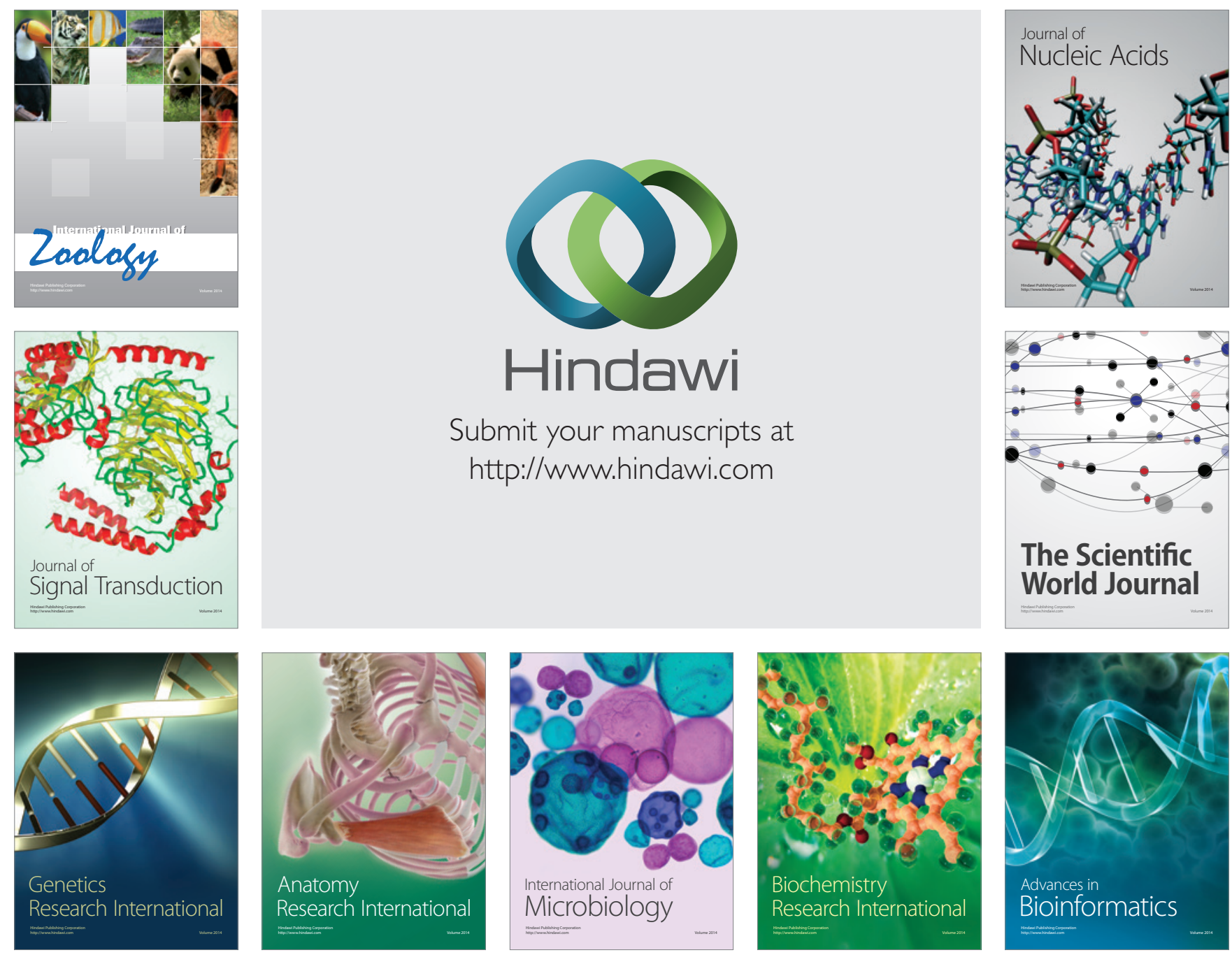

The Scientific World Journal
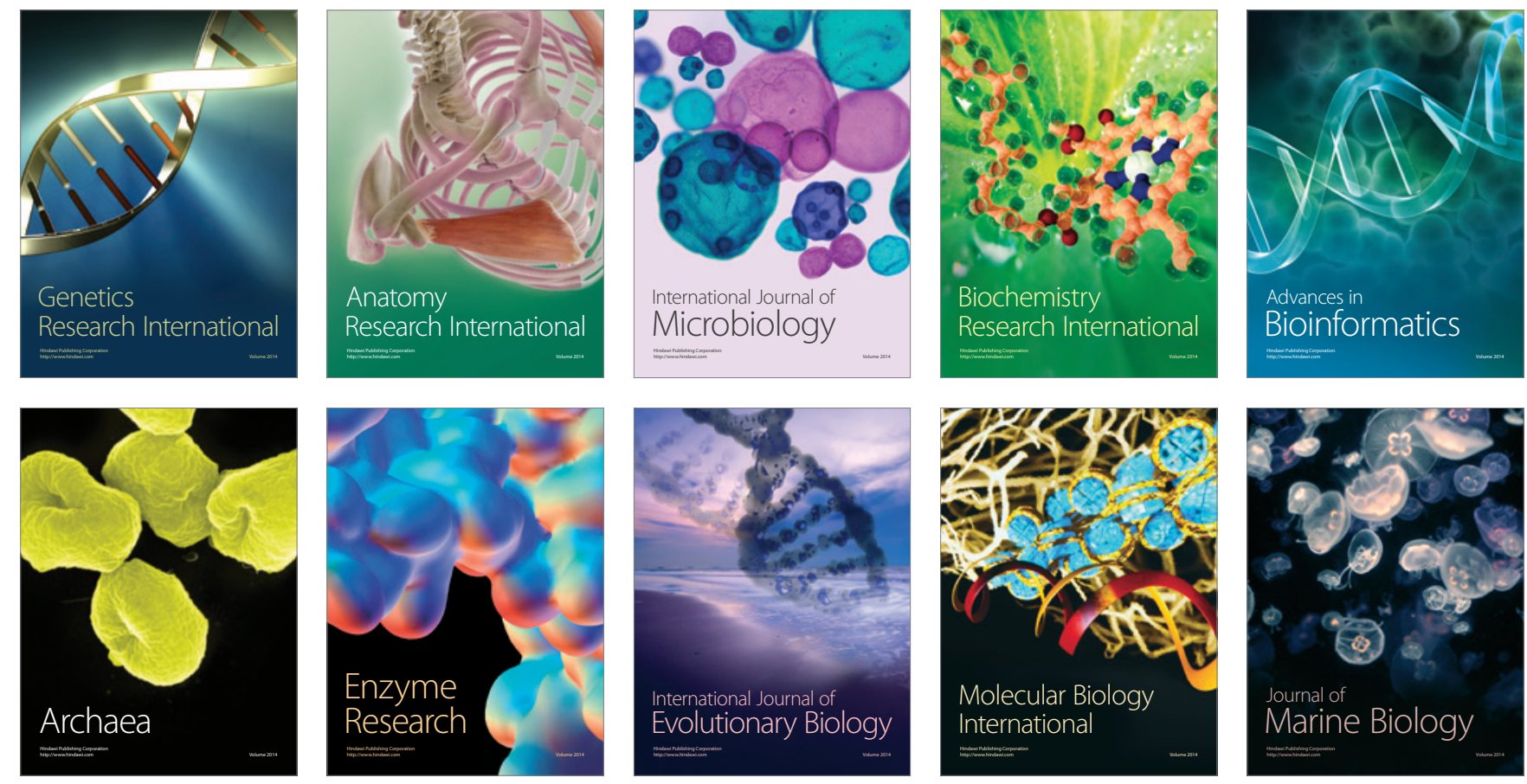